\title{
Universe: the dynamics of the minimum and maximum expansion states
}

Sócrates Petrakis ( $\square$ sgeorgespp@gmail.com )

S.E.E.-M.G. https://orcid.org/0000-0001-7549-1009

Physical Sciences - Article

Keywords: Universe, Energy, Spin, Gravity, Entropy

Posted Date: October 28th, 2021

DOI: https://doi.org/10.21203/rs.3.rs-708548/v14

License: (c) (i) This work is licensed under a Creative Commons Attribution 4.0 International License. Read Full License 


\title{
Universe: the dynamics of the minimum and maximum expansion states
}

Sócrates Georges Petrakis

SEEMG, Belo Horizonte - MG, Brazil

Physicist Licensed (UFMG)

Research Square (DOI): https://www.researchsquare.com/article/rs-708548/v1

Inspire: http://inspirehep.net/record/1894962

ORCID ID: 0000 - 0001 - 7549 - 1009

E-mail: sgeorgespp@gmail.com

E-mail: socrates.petrakis@educacao.mg.gov.br

Versão (português): https://www.universocompleto.org/

\begin{abstract}
Two hypotheses stand out in describing the evolution of the Universe. The predominant one predicts that the current expansion began at a certain instant and will not preserve any variation of energy that performs work; apparent flat Universe $(\Omega=1)$ is advocated by relativistic calculations and observational data, with an end or thermal death at its maximum expansion (3D Space). The other hypothesis considers that the Universe is cyclical (always alternating phases of expansion and contraction). This proposal aims to demonstrate that both hypotheses can be correct by not being distinct, but complementary. Supported by the immutability of physical laws, analyses of concepts such as space, mass, energy, gravity, spin, and entropy define an exclusive presence of 1D Space in the minimum and maximum expansion states of the Universe. With our 3D Space Universe created and existing between these extreme states, every dynamic is outlined and completes the usual relativity. The concept of complete rest energy (1D Space) was able to be applied, demonstrating that the complete evolution of the Universe is spatially dynamic in a perpetual time dimension, always recreating our Universe.
\end{abstract}

Keywords: Universe, Energy, Spin, Gravity, Entropy. 


\section{Introduction}

The state of the Universe depends on energy. If the Universe is in an extremely contracted state, with the scarcity of space inside, no environment that promotes life would exist. In contrast, if the Universe is in a well-expanded state, the energies would not generate agglutinations for planetary formations or existence of life; therefore, the study of universal evolution [1] is important.

In Cosmology, the evolution of the Universe [2] is studied via astronomical observations, particle collision experiments, computational analysis, and

relativistic calculations. However, its complete understanding is hindered by factors, such as the unknown nature of some of its constituents.

The most accepted theory about our Universe evolution posits a certain initial state and an accelerated spatial expansion. However, the structure generating this expansive beginning is still under debate.

The instants of extreme universal contraction are usually related to presence of a great energy concentration.

Moreover, the existence of an expansive universe, the predicted current amount of mass, and entropy (S) are commonly associated with a future thermal death or total dissipation.

Given these current associations, this study highlights concepts such as energy [3], dimension, and Universe to present solutions in a coherent development.

\section{Development}

The Universe can be considered the holder of all space-time, that is, any massive or energy content which results in a space variation related to the time dimension [4].

The characterization of the true extreme states is necessary to define a complete evolution of the Universe. Already well-established physical concepts can facilitate the achievement of this objective. 
For a complete analysis of the whole evolution, it should be considered that conceptually, the Universe must be in a static state (finite) of maximum contraction to initiate an expansion. Just as the minimum temperature cannot be lower than Absolute Zero, the most extreme contraction will be limited to the maximum possible absence of space; for this extreme state, the universe holds a finite amount of energy which represents its contraction, and which will also be available to represent the maximum state of expansion in a possible conversion.

Complete evolution limited in extreme states:

For the beginning or the end of any relative motion, it is necessary to be at rest.

Before the expansive beginning, the entire universe had to be at rest.

For the maximum expansion, the entire Universe will be at rest.

For the extreme and static states, conserving the dynamic, the existence of maximum potential energy is necessary, i.e., the presence of a maximum distance (occupied or not) or maximum 1D Space.

During a complete evolution, the possible spatial variation is always the same: limited by 1D (extreme states).

The entropy depends on the spatial distribution of the components or on the degree of freedom present, that is, on the type of spatial dimension that is existing: 3D, 2D or 1D; the degree of freedom of complete universal evolution is limited to the 1D spatial dimension.

Maximum contraction: the 1D spatial dimension has a maximum occupation; relative motion is zero; spin is zero.

Maximum expansion: the 1D spatial dimension has a minimum occupation; the space (between the two minimum 1D particles) is maximum; spin is zero.

There are studies that propose the exclusive existence of lower dimensions [5, 6]. 
Relativity (3D + 1D) and quantum mechanics analyze the beginning of the expansion only of our Universe (3D Space); the initial instant of expansion of the complete universal evolution happened earlier, in 1D spatial dimension.

Since our 3D space dimension in a partial evolution of the universe does not conserve its total energy (relativity), a complete evolution may indicate conservation of energy.

When the internal energetic interactions of any amount of rest mass $\left(\mathrm{m}_{0}\right)$ are undone, all value converted of gravitational potential energy $(\mathrm{Eg})$, of energetic interactions: quantum, atomic and electromagnetic (Eq), and of internal kinetic energy (Ek) [7] are equivalent to the total rest energy $\left(E T=E_{0}=m_{0} \times c^{2}\right)[8]$.

The total invariant "mass" (at the resting instant of the universe: minimum or maximum expansion) is the analog of the resting "mass".

Thus, ET (Exclusive 1D Spatial Dimension) is expressed as follows:

$$
\mathrm{ET}=\mathrm{E}_{0}=\mathrm{Eg}+\mathrm{Eq}+\mathrm{Ek}=\mathrm{m}_{0} \times \mathrm{c}^{2}
$$

For Universal resting state (minimum and maximum (1D) expansion), the following points are considered:

- $\mathrm{ETmaxC}=\mathrm{EgC}+\mathrm{EqC}+\mathrm{EkC}$, where $\mathrm{ETmaxC}, \mathrm{EgC}, \mathrm{EqC}$ and $\mathrm{EkC}$ are the total energy, potential energy (due to the presence of 1D space "mass"), Eq, and Kinetic energy, respectively, in the state of maximum contraction.

- $\mathrm{ETmax}=\mathrm{EgE}+\mathrm{EqE}+\mathrm{EkE}$, where $\mathrm{ETmax}, \mathrm{EgE}, \mathrm{EqE}$ and $\mathrm{EkE}$ are the total energy, potential energy (due to the presence of 1D space "mass"), Eq, and Kinetic energy, respectively, in the state of maximum expansion.

- $E k C=0$ (for the resting state of maximum contraction, the relative motion is zero).

- $E k E=0$ (for the resting state of maximum expansion, the relative motion is zero). 
- $\mathrm{EqC}=0$ (In a completely static state: $E k C=0$. Quantum energy $(\mathrm{Qe})=$ Planck constant $(\mathrm{h}) \times$ Frequency $(\mathrm{F})=0(\mathrm{~F}=0)$. There is no atomic presence and no electromagnetic interactions).

- $\mathrm{EqE}=0$ (In a completely static state: $\mathrm{EkE}=0 . \mathrm{Qe}=\mathrm{h} \times \mathrm{F}=0(\mathrm{~F}=0)$, no atomic presence, and no electromagnetic interactions).

- $\mathrm{ETmax} E=\mathrm{ETmaxC}=\mathrm{EgE}($ maximum value $)+\mathrm{EqE}(=0)+\mathrm{EkE}(=0)=$ $\mathrm{EgC}$ (maximum value) $+\mathrm{EqC}(=0)+\mathrm{EkC}(=0)$.

- For maximum $\mathrm{EgE}$, the maximum distance (the space) between the two minimum "massive" particles (one- dimensional) must be present.

- For $\mathrm{EgC}$, the maximum value must be present, even with the scarce spaces between the "masses".

- For EgC to have the maximum value, the maximum amount of "mass" must be distributed over a length of one dimension (maximum distance between centers of "mass").

If for EgC the masses are distributed in 1D length $(d=2 r)$, and for EgE the maximum distance (the space) between the two minimum "massive" particles is $1 \mathrm{D}$, the gravitational field is uniform, and the reference point is the center of mass.

For these extreme states, the use of the integral: $U(x)=\int F(x) d x=$ $\mathrm{Eg}=(-\mathrm{G} . \mathrm{m} . \mathrm{m} / \mathrm{r})$ makes the value of the potential energy negative, i.e., Eg has a maximum value tending to zero, in the static and extreme 1D spatial states (with Ek =0).

With a uniform gravitational field (1D), Eg can be maximum and positive, $\mathrm{Eq}=0$ and $\mathrm{Ek}=0$. 
For the minimum and maximum expansion of the Universe, the following points are considered:

- The capacity to do work of the extreme states or the possible energy variation (conversion) is always the same, limited by the 1D spatial dimension or perpetual existence of some space between the "masses" (energy variation).

- Maximum expansion: two minimum "masses" (particles) are separated to maximum by $1 \mathrm{D}$ space, corresponding to $\left.\mathrm{E}_{0}=\mathrm{ETmax}=\mathrm{EgE}\right)$.

- Maximum contraction: two minimum spaces are present (the opposite state from the state of maximum expansion), i.e., the universal structure is composed of three "mass" parts: one minimum "mass" (particle) between two maximum "mass" $\left(\mathrm{m}_{0}+\mathrm{m}_{0}\right)$ for the conservation of energy.

- $M_{0}-m_{0}=$ Minimum "mass" (1D particle; where $M_{0}>m_{0}$, and $\left.M_{0} \cong m_{0}\right)$.

- $\mathrm{M}_{0}+\mathrm{M}_{0}+\left(\mathrm{M}_{0}-\mathrm{m}_{0}\right)=$ Universe total "mass" in the maximum contraction state (if it were possible the non-existence of two minimum spaces).

- The energy of the maximum contraction state (1D Space) is the gravitational potential energy related to the "masses" $\left(M_{0}+M_{0}+\left(M_{0}-m_{0}\right)\right)$ subtracted from $2\left(M_{0}-m_{0}\right)$ that would correspond to the two minimum "masses" that could occupy the two present spaces.

- There is no energy directly associated with the existence of the spaces (1D) in these extreme states of the universe. Such existence is related to a limit of gravitational potential energy (existence of a determined amount of mass) in states of maximum, but not complete, contraction or expansion.

- $m_{0}+m_{0}+\left(M_{0}-m_{0}\right)=$ Universe total "mass" in the maximum contraction state (with the necessary presence of the two minimum spaces). 
- Energy available in the maximum contraction state:

$\left(M_{0}+M_{0}\right) \times c^{2}+\left(M_{0}-m_{0}\right) \times c^{2}-2\left(M_{0}-m_{0}\right) \times c^{2}=$

$\left(m_{0}+m_{0}+\left(M_{0}-m_{0}\right)\right) \times c^{2}=\left(M_{0}+m_{0}\right) \times c^{2} ;$

with the necessary presence of two internal spaces, corresponding to an incomplete but maximum contraction for the conservation of energy:

$\mathrm{E}_{0}=\mathrm{EgC} \neq 0$.

- The energy of the state of maximum expansion is the gravitational potential energy related to the presence of space (1D), or the absence of "masses": $\left(M_{0}+M_{0}+\left(M_{0}-m_{0}\right)\right)$, subtracted from the presence of two particles $\left(2\left(\mathrm{M}_{0}-\mathrm{m}_{0}\right)\right)$ at the extremities that represent the absence of space (empty) or expansion.

- Energy available in the maximum expansion state:

$\left(\left(M_{0}+M_{0}\right) \times c^{2}+\left(M_{0}-m_{0}\right) \times c^{2}-2\left(M_{0}-m_{0}\right) \times c^{2}\right)=$

$\left(\left(M_{0}+M_{0}\right) \times c^{2}-\left(M_{0}-m_{0}\right) \times c^{2}\right)=\left(M_{0}+m_{0}\right) \times c^{2}$

Two minimum "massive" particles separated to maximum by a space

(1D) corresponding to incomplete and maximum expansion, for the energy conservation, $\left(\mathrm{E}_{0}=\mathrm{EgE} \neq 0\right)$.

In this way, the extreme states are represented as follows:

Energy available: $\mathrm{ETmaxC}=\mathrm{E}_{0}=\mathrm{EgC}=\left(\mathrm{M}_{0}+\mathrm{m}_{0}\right) \times \mathrm{c}^{2}$

Energy available: $\mathrm{ETmax} E=\mathrm{E}_{0}=\mathrm{EgE}=\left(\mathrm{M}_{0}+\mathrm{m}_{0}\right) \times \mathrm{c}^{2}$

$E T \max C=E T m a x E$ (conservation of the energy available)

$\left(M_{0}+m_{0}\right) \times c^{2}=\left(M_{0}+m_{0}\right) \times c^{2} \quad($ Energy available $)$

ETmaxE $=\left(M_{0}+m_{0}\right) \times c^{2}$, does not mean that the universe will have a rest "mass" quantity $\left(\mathrm{M}_{0}+\mathrm{m}_{0}\right)$ in its state of maximum expansion, but that it will have an energy corresponding to the universe at maximum contraction (the state with this rest mass present). 
Kinetic energy is zero in linear and static states; although there is no more curvable space in these states, gravitational potential energy cannot convert instantaneously into kinetic energy throughout the universe; the conversion occurs from the beginning of motion.

In extreme states (1D Space), not only Time, but also Space is a linear dimension.

The entropy (S) or the degree of freedom must be associated with the dimension; the degree is the same in extreme states of the 1D Spatial Dimension ( $\Delta S=0$ ); therefore, entropy is minimum (the degree of freedom is minimum), at minimum and maximum expansion; thus, expansions are followed by contractions of the same intensity.

Figs. 1-3 show (sketch) that any contraction and expansion, although intense, cannot be total; demonstrating that the Universe is dynamic, always transforming with the preservation of some energetic variation.

Figure 1. The entire Universe (complete evolution).

\section{Entire Universe:}

All existence $=$ Masses (energies) + space between masses)

Entire Universe (maximum expansion,1D Space)

(Non-existence) .

(Our Expanding 3D Space Universe)

(The space that can exist by maximum expansion) 
Figure 2. Universe (1D maximum contraction, 2D, 3D, inflation, 2D, 1D maximum expansion)

Expansion: creates space by the interaction of pre-existing masses of 1D, 2D, or 3D internal space.

\section{Maximum Contraction}

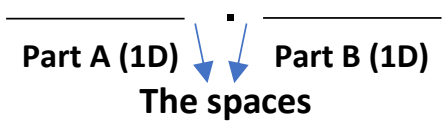

For maximum contraction: maximum EgC (maximum "mass" (1D) and distribution in 1D Space, maximum distance between centers of mass with occupied space); $\quad E q C=0 \quad$ and $\quad E k C=0$; Spin $=0$. Relative motion $=\mathrm{V}=0$.

Maximum concentration for minimum unoccupied (between masses) space.

Part A (mo), Part B (mo), particle (Mo - mo) and only two spaces (1D) exist $=$ no spatial curvature $(E k C=0)$ $=$ maximum Eg starts to decrease $=$ Ek increases (energy conservation).

No spatial curvature: the expansive process starts.

Until all possible 1D and 2D "mass" is converted into 3D mass (internal space), the total amount of mass (1D +2D + 3D) will be conserved.

\section{D Spatial Dimension expanding:}

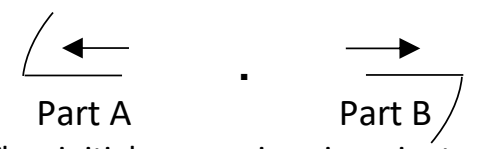

The initial expansion is oriented according to the fact that the disappearance of any curvature from the center is inferred to the extremities as the expansion progresses.

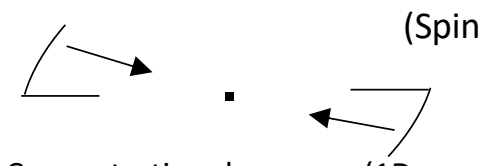

Concentration decreases (1D spatial dimension); Eg (potential) decreases (1D to 2D spatial dimension); Ek and spin increase.
There is space between the "masses" (Part A and B) and the central particle [9].

Eg continues to decrease with the formation of an edge = approximation of the parts $=$ Ek (spin) increases (1D to 2D spatial dimension).

The total amount of (maximum) mass is preserved, and the 2D dimensional internal space is larger than the (previously existing) 1D dimensional space.

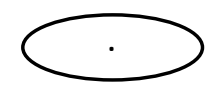

Spin 2D (Space):

From the 1D spatial dimension (with maximum "mass") to the 2D spatial dimension = Expansion.

The interactions in the edge (Presence of the central particle) start to form particles with 2D internal space $=$ filling the 2D internal space (Universe).

With the total amount of mass being conserved, and the amount of 2D "masses" increasing to the maximum possible, the 2D "mass" concentration increases (in 2D space), the spin increases, generating more intense centrifugation $=$ expansion .

Occupied by 1D and 2D Internal space component :

2D space

With many 2D particles in the 2D spatial dimension, the interactions continue, forming the first particle of internal 3D space (when starting the expansion into 3D space).

\section{Spin 3D Space:}

Our Universe 3D space (expanding): "point" (3D) + ("field")

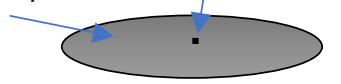

Our Universe of 3D space emerges, formed by 3D particle (point, "Boson"), 1D and 2D particles "Field".

The initial 3D mass formation depends on the non-flattening of the space by centrifugation = "mass" (energy) concentration = mass 3D (" $\left.E_{0}=m_{0} \times c^{2}{ }^{\prime \prime}\right)=$ "excitation" of the field from $2 D$ to $3 D$ by curvature in the central space.

With interaction, the amount of 1D and 2D particles ("masses") decreases while the amount of 3D masses increases

The total amount of mass remains preserved.

With a higher concentration of $3 D$ mass in $3 D$ space, spin and centrifugation are intensified = inflation. Inflation:

$3 \mathrm{D}$ space with high spin $=$ flat universe

(Flattened)

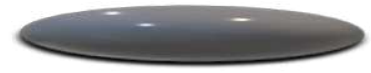

After inflation, the amount of $1 D$ and 2D particles continues to decrease, while the amount of 3D mass increases. The concentration of $3 D$ mass in 3D space increases (spin increases, Ek and $\mathrm{Eq}$ increases, and $\mathrm{Eg}$ decreases). The expansion of the Universe will be accelerated with the increase of its spin (3D), and 3D mass (by centrifugation).
There are studies that propose a flat, or a closed Universe [10]. The extreme linear states of the space are the that define the continuity of evolution.

The dark (matter and energy) can represent 1D or 2D particles, which when they interact ("excitation" of the field) and form more massive particles (3D internal space), increase the speed of spin and accelerate the expansion of our Universe (3D space dimension).

After the maximum formation of $3 D$ masses in 3D space, the expansion begins to slow down.

With the 3D mass tending to dissipate (continued expansion), the concentration in 3D space decreases, EK and the intensity of the spin decreases = Eg increases.

Future of expansion: 2D Spatial Universe, completely flat (2D).

(Expanding Disk)

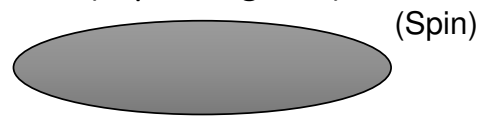

The 3D spatial dimension becomes 2D Space (Disk); spin and EK decrease, Eg (potential) increases, with less and less mass.

For maximum expansion, the minimum particles 1D "masses" emerges:

2 (Mo - mo), separated by the maximum space:

$\left(\mathrm{EgE}=\left(\mathrm{M}_{0}+\mathrm{m}_{0}\right) \times \mathrm{c}^{2}\right)$

$$
\text { Linear Space (1D) }\left(\mathbf{M}_{\mathbf{0}}-\mathbf{m}_{\mathbf{0}}\right) \quad\left(\mathbf{M}_{\mathbf{0}}-\mathbf{m}_{\mathbf{0}}\right)
$$

The universe does not continue to expand (the existence of two minimum particles with "mass" (1D) preserves the total energy):

$(\mathrm{ETmaxC}=\mathrm{ETmax}$ ). $\mathrm{EkE}=0$; $\mathrm{EqE}=0 ; \mathrm{EgE}$ (Maximum). Due to the absence of curvable space, Eg decreases with the return of the motion. 
Figure 3. Universe (1D maximum expansion, 2D, 3D, 2D, 1D maximum contraction)

Contraction: Eg starts to decrease and
Ek to increase with minimum pre-existing
"mass". Mass generated by spatial
curvature.

Maximum expansion state: In a linear dimension, without Curvature of space, $\mathrm{Eg}$ decreases $=\mathrm{Ek}$ increases. For conservation of energy, the existing particles cannot dissipate or expand. The only possible motion for Ek to increase is the start of contraction. 1D Spatial dimension (with minimum "mass") to 2D Spatial dimension = contraction.

The initial contraction is oriented according to the fact that the signal from the complete disappearance of the curvature of space does not instantaneously reach the entire universe; Eg does not convert to Ek instantaneously.

The contraction begins: With a change in energy (conversion of Eg to Ek):

$$
\begin{aligned}
& \text {. } \longrightarrow \text { (v) } \\
& \left(M_{0}-m_{0}\right) \\
& \text { (v) } \longleftarrow \text {. } \\
& \left(M_{0}-m_{0}\right)
\end{aligned}
$$

Speed (v) increases until it tends to the limit (c).

Just as the Relativity of time varies according to the speed and gravity present, the spatial dimension in extreme states is guided by the existing gravitational potential and kinetic energy.

The possible variation of Time relativity is always the same: limited by speed $(v=0$ and $\mathrm{v}=\mathrm{c}$ ).

Limited variation in time relativity $\leftrightarrow$ "mass" (or energy concentration) limited in 1D, 2D or 3D space $\leftrightarrow$ limited contraction and expansion. When $v$ tends to $c$, the only way to complete the conversion process from $\mathrm{Eg}$ to $\mathrm{Ek}$ is to generate 2D "mass". Concentration for mass formation happens more easily in 1D or 2D Space because of the lack of space (3D).

$$
(\mathrm{v} \cong \mathrm{c}) \longrightarrow(\mathrm{v} \cong \mathrm{c})
$$

As the 2D "mass" is formed, there is a tendency for the speed (v) to decrease at an edge or orbit in a curved 2D Space. While the conversion (Eg to Ek) continues, (v) is restored. Thus, the 2D "masses" are formed in 2D space.

2D Space(Spin)

\section{The contraction continues:}

Total amount of mass (1D+2D) internal space) increase $=$ increases concentration (2D spatial dimension) and spin. Eg has not yet fully converted to Ek and Eq.

The maximum possible amount of mass that will be produced has not yet been realized. The spatial curvature and concentration increase until they form 3D masses with the $3 \mathrm{~d}$ dimension.

When mass production ends, the total mass amount, concentration, and spin become maximum (3D spatial dimension), the contraction starts to decelerate. Ek starts to decrease and Eg starts to increase. maximum mass amount and spin (3D dimension):

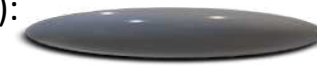

Flat Universe

The contraction continues: Ek has not yet fully converted to $\mathrm{Eg}$.

\section{(3D to 2D and 1D Space):}

Via centrifugation and planarization (Ek starts convert into $\mathrm{Eg})$.

2D disk + two particles arise. This incomplete contraction to 2D will consist of a fully compressed disc, between two particles (symmetry), so that there are spaces, for the presence of potential energy (maximum "mass" of the disk between the two particles).

(Spin decreases)

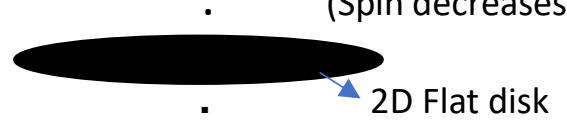

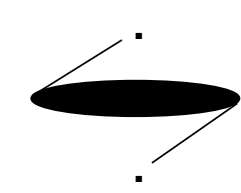

The spin (centrifugation) allows the existence of space for motion, only between the edge of the disk and the particles, i.e., the existing space has a double cone shape. This shape (by equilibrium) does not allow the two particles to approach in the direction of the disk. In its maximum state of contraction 2D, the disk (without internal spaces) loses its internal gravitational effect, the contraction continues (spin) to 1D spatial dimension.

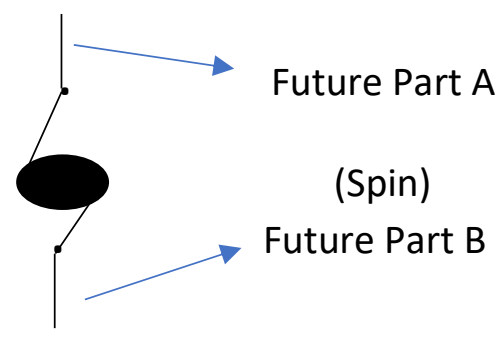

The disk decreases from the edges with the spin, and through the only space that exists: the double conical space. The homogeneous distribution of "mass" from the disk to the future parts ( $A$ and $B$ ) is defined by spin and entropy.

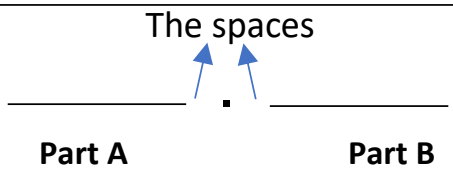

(Ek completely converts to $\mathrm{Eg}(\mathrm{EgC})$ with maximum "mass").

Consecutive new expansion:

Through linearization, the universe becomes a 1D spatial dimension. The universe returns to the state of maximum contraction (with maximum "mass"), and the parts are separated by more necessary central spaces and a minimum particle (which allows conservation of the total 1D energy). Parts A, $B$, and the particle completely reach 1D spatial dimension, and the expansion resumes. $\Delta S$ (extreme states) $=0$. 


\section{Conclusion}

The Big Bang is a well-established theory with the application of relativity and the use of observational data, effectively describing the evolution of our 3D spatial Universe, and allowing for a possible application of quantum mechanics in an initially dynamical environment.

Similarly, well-established concepts preclude the description that something can expand from nothing, or that any expansion can proceed indefinitely from an infinite past. By expansion is assumed a maximum contraction instant that is the minimum existence of space. Just as there is no temperature below Absolute Zero, there can be no state of contraction more intense than the one that already has the minimum possible space.

Thus, to initiate a motion of expansion, the universe must necessarily be in a state of maximum contraction and static.

Support for this proposal is based on the clearest presentation of an evolution that respects the Big Bang theory, without conflicting with some basic concepts of physics.

Mathematics is partially restricted to our $3 \mathrm{D}$ or non-static Universe, in presenting exclusive 1D and 2D spatial dimensions.

It has been demonstrated that there can be maximum contraction to a single spatial dimension, but not so intense to be complete; similarly, maximum expansion (1D Space between "masses") can only occur without the complete dissipation, preserving an eternal existence.

The fact that the sign of the complete disappearance of the curvature of space in the extreme states of universal evolution does not instantaneously reach the entire Universe is related to the concept of existence, space, and time. 
Experiments with neutrinos (2020) show the possible asymmetry in the formation of matter and antimatter [11]. Antimatter formation may prevail in the contraction phase of the Universe, and thus symmetry may occur only in the contraction + expansion set.

The description of a contraction and an expansion extended to a single spatial dimension was necessary to delineate a more complete evolution with 1D spatial Universe that generates the dynamics in truly extreme and static states, where there is no scope for vibrations or oscillations. The presence of $1 D$ and $2 D$ "masses" interacting and increasing the 3D massive presence was proposed, explaining the current accelerating expansion in 3D space.

The mass distribution and the degree of freedom depends on the amount of mass, and on the existing space (between these). Thus, in a space of maximum 1D spatial contraction or maximum 1D spatial expansion, the degree of freedom or entropy is minimum.

Energy (of a complete Universal evolution) is neither created nor lost; energy is exchanged or transformed. The current motion of the Universe already represents an energetic and spatial variation.

The rest energy $\left(E_{0}\right)$ in 1D (Space) was considered, thus ruling out the use of certain relativistic and quantum calculations $(4 \mathrm{D}=3 \mathrm{D}($ Space $)+1 \mathrm{D}($ Time $))[12,13]$.

The Dynamic Universe has an eternal transformation of energy; contraction and expansion are limited, but in continuous cycles. Energy is limited by the 1D space dimension by the necessary presence of empty spaces at maximum contraction, and minimum "masses" at maximum expansion. 
For the beginning or end of any motion (expansion), the entire universe must be at rest. Relative motion is zero only in the states and at the instant of maximum contraction and expansion. Such instants have energy that can be transformed and sustain motion in subsequent times, preserving existence.

Equation has been formulated that respects the necessary preservation of total energy in the complete evolution Universal: $\left(M_{0}+m_{0}\right) \times c^{2}=\left(M_{0}+m_{0}\right) \times c^{2}$

In the extreme states of 1D spatial dimension, all the forming components, make up the one mass (the Universe) at complete rest, with the inherent presence of space between the constituents. The model presented can demonstrate why the Universe begins its spin with the onset of expansion [9].

In our Universe (3D spatial dimension), the mass that is directly perceptible is formed by three-dimensional (3D) internal spaces. "Mass" formed by internal spaces (2D) is existence in which one of its component spatial dimensions is minimum, or without space for variation; "mass" formed by internal spaces (1D) is existence in which two of its dimensions are minimum, without space for variation.

The smallest "massive" particles (formed by internal spaces 1D or 2D) and their associated energies are occupying and propagating in our universe (3D Space). while these particles do not bond to form 3D internal space particles, their presences are perceived by the gravitational effect of their existence. When they group together to form 3D particles, they may represent our accelerated expansion, by the faster spinning of our 3D (Space) universe.

Every energy signal is absorbed in the maximum contraction state of each cycle; thus, Olbers' paradox [14] can be disregarded.

This approach respects the Ockham razor perspective and the principle of parsimony. The best explanation should assume the fewest number of premises [15]. 


\section{References}

[1] Universe. Webster`s New World College Dictionary. Wiley Publishing, Inc. [S.I.: s.n.]. 2010

[2] Souza Y.L. Cosmologia (Cosmology). Infoescola (2018)

[3] Baptista J.P.: Os princípios fundamentais ao longo da história da física. Rev. Bras. Ensino Fís. 28, 541-553 (2006)

[4] Zeilik, Michael; Gregory, Stephen A. Introductory Astronomy \& Astrophysics (4⿳亠丷厂 ed.). Saunders College. ISBN 978-0-03-006228-5 (1998)

[5] Mureika J., Stojkovic D.: Detecting Vanishing Dimensions via Primordial Gravitational Wave Astronomy. Physical Review Letters, Vol.: 106, 101101, March (2011)

[6] Anchordoqui L., Dai De Chang, Fairbairn M., Landsberg G., Stojkovic D.: Vanishing Dimensions and Planar Events LHC. arXiv: 1003,5914. (2011)

[7] Jain C.M.: Textbook of engineering physics. S.I.: Phi learning (2009)

[8] Vieira S., Barros A., Araújo I., Oliveira J.C.T.: A comparison among deductions of the equation $\mathrm{E}=\mathrm{m} \mathrm{c}^{2}$. Rev. Bras. Ensino Fís. 26, 93-98 (2004)

[9] Wang, P. Libeskind, N.T., Tempel, E.et al. Possible observational evidence for cosmic filament spin. Nat Astron (2021).

[10] Valentino, E.D., Melchiorri A., Silk J.: Planck evidence for a closed Universe and a possible crisis for cosmology. Nature Astronomy 4, 196 - 203 (2020)

[11] The TK Collaboration, Constraint on the matter-antimatter symmetry-violating phase in neutrino oscillations. Nature. 580, 339-344 (2020)

[12] O'Connor J.J., Robertson E.F.: General relativity. Mathematical Physics Index. 1996. Esc. Mat. Estatística Univ. St. Andrews (1996)

[13] Roberto B., Netto L.F.: What is the special theory of relativity. Science meeting. (O que é a Teoria da Relatividade Especial). Feira de Ciências (2009)

[14] Souza R.E.: Introduction to cosmology (Introdução à Cosmologia). São Paulo, Edusp (2004)

[15] Ariew R.: Ockham's razor: a historical and philosophical analysis of Ockham's principle of parsimony. Champaign-Urbana, University of Illinois (1976) 